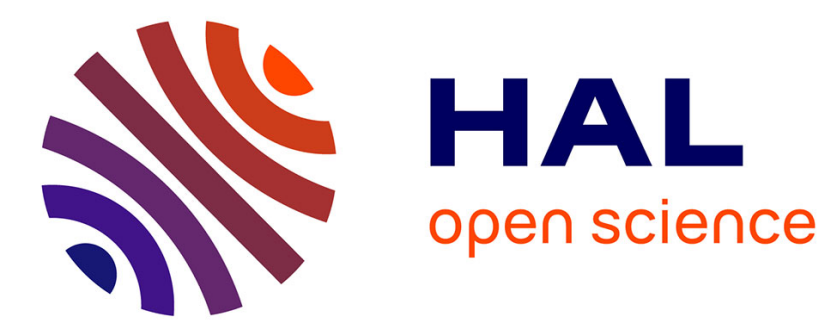

\title{
Ultrafast ultrasound imaging grants alternate methods for assessing diaphragm function
}

\author{
Damien Bachasson, Martin Dres, Marie-Cécile Nierat, Jonne Doorduin, \\ Jean-Luc Gennisson, Jean-Yves Hogrel, Thomas Similowski
}

\section{- To cite this version:}

Damien Bachasson, Martin Dres, Marie-Cécile Nierat, Jonne Doorduin, Jean-Luc Gennisson, et al.. Ultrafast ultrasound imaging grants alternate methods for assessing diaphragm function. IEEE International Ultrasonics Symposium (IUS 2013), Jul 2013, Prague, Czech Republic. 10.1109/ULTSYM.2018.8579781 . hal-03275175

\section{HAL Id: hal-03275175 \\ https://hal.science/hal-03275175}

Submitted on 30 Jun 2021

HAL is a multi-disciplinary open access archive for the deposit and dissemination of scientific research documents, whether they are published or not. The documents may come from teaching and research institutions in France or abroad, or from public or private research centers.
L'archive ouverte pluridisciplinaire HAL, est destinée au dépôt et à la diffusion de documents scientifiques de niveau recherche, publiés ou non, émanant des établissements d'enseignement et de recherche français ou étrangers, des laboratoires publics ou privés. 


\title{
Ultrafast ultrasound imaging grants alternate methods for assessing diaphragm function
}

\author{
Damien Bachasson ${ }^{1}$, Martin Dres ${ }^{2}$, Marie-Cécile Nierat ${ }^{2}$, Jonne Doorduin ${ }^{3}$, Jean-Luc Gennisson ${ }^{4}$, Jean-Yves \\ Hogrel $^{1}$, Thomas Similowski ${ }^{2}$ \\ ${ }^{1}$ Institut of Myology, PARIS (France) \\ ${ }^{2}$ Sorbonne Université, INSERM, UMRS1158 Neurophysiologie Respiratoire Expérimentale et Clinique; AP-HP, \\ Groupe Hospitalier Pitié-Salpêtrière Charles Foix, Service de Pneumologie et Réanimation Médicale du \\ Département R3S - PARIS (France) \\ ${ }^{3}$ Department of Neurology and Clinical Neurophysiology, Donders Center for Neuroscience, Radboud University \\ Medical Center - NIJMEGEN (Netherlands) \\ ${ }^{4}$ IR4M, CNRS UMR 8081, Université Paris-Saclay, CEA Service Hospitalier Frédéric Joliot, ORSAY (France)
}

\begin{abstract}
The diaphragm is the main respiratory muscle. Availability of accurate and noninvasive methods for the assessment of its function is critical. In this work, we propose two techniques based on plane wave imaging (PWI) and shear wave elastography (SWE) for assessing diaphragm contractility. The first technique uses PWI to image short responses ( 200 ms) evoked by artificial stimulation of the phrenic nerve(s). The second technique use SWE to monitor change in diaphragm stiffness during continuous ventilation and/or respiratory maneuvers. Preliminary results from experiments performed healthy participants are presented. Our data showed that SWE and PWI can provide a new opportunity for direct and non-invasive assessment of diaphragm function.
\end{abstract}

Keywords - diaphragm, ultrafast ultrmù !asound imaging, shear wave elastography, plane wave imaging

\section{INTRODUCTION}

The diaphragm is the main respiratory muscle. Gold standard methods for assessing diaphragm function involves the recording of transdiaphragmatic pressure (Pdi) or electromyographic response gathered during ventilatory tasks or in response to artificial stimulation. Pdi computation requires the measurement of esophageal and gastric pressures using internal probes introduced in the nose. Surface electromyography of the diaphragm is noninvasive but exhibits limited validity for assessing diaphragm activity. Both Pdi and electromyography are indirect measures of diaphragm contractility [1]. Ultrafast plane wave imaging (PWI) enables very high frame rate that allows capturing very short stimulated muscle contractions ( 100 ms) [2]. PWI also allows shear wave elastography (SWE) that has been shown to provide reliable estimates of force output in locomotor muscles [3]. We propose two key techniques based on PWI and SWE for assessing diaphragm contractility. The first technique uses PWI to image short responses $(\sim 200 \mathrm{~ms})$ evoked by artificial stimulation of the phrenic nerve(s). The second technique use SWE to monitor change in diaphragm stiffness during continuous ventilation and/or respiratory maneuvers. The current work introduces these techniques and preliminary data from healthy humans are also provided.

\section{ULTRAFAST PLANE WAVES FOR IMAGING DIAPHRAGM RESPONSE TO PHRENIC NERVE STIMULATION}

Ultrasound imaging is commonly used to measure excursion and/or thickening of the diaphragm during ventilation or during voluntary respiratory maneuvers [4]. However, non-volitional assessment of diaphragm function is critical to specifically evaluate diaphragm contractility and to decipher the causes of diaphragm dysfunction. Unilateral or bilateral electrical or magnetic stimulation of the phrenic nerve(s) may be used to elicit non-volitional contractions of the diaphragm. The amplitude of the Pdi response to stimulation is considered as the most specific manner to investigate diaphragm contractility. The delay and shape of the electromyographic response of the diaphragm may also be studied to assess nerve conduction velocity and diaphragm contractility, respectively [1]. Artificial stimulation is usually performed using single pulses so that responses are typically within the $100-200 \mathrm{~ms}$ range. Imaging such responses cannot be achieve using conventional focused ultrasound imaging approaches that display largely insufficient frame rates $(\sim 100 \mathrm{~Hz})$. Ultrafast PWI may be used to capture movies of such events.

\section{SHEAR WAVE ELASTOGRAPHY FOR ASSESSING DIAPHRAGM CONTRACTILITY DURING CONTINUOUS VENTILATION OR RESPIRATORY MANEUVERS}

Given that shear wave elastography is based on the measurement of shear wave speed induced in the medium by ultrasound beams [5], it cannot be used to monitor responses induced by single pulse stimulation. In addition, diaphragm thickening fraction measured using conventional ultrasound imaging exhibit limits for quantifying diaphragm effort [6]. However, SWE may be used during longer event such as continuous ventilation and/or respiratory maneuvers.

\section{MATERIEL \& METHODS}

Fourteen healthy volunteers were studied. All participants gave written informed consent. This study conformed to the Declaration of Helsinki and was approved by the local ethics committee. Diaphragm ultrasound imaging was performed using an ultrafast ultrasound scanner (Aixplorer, Supersonic Imagine, France) driving a 10-2 MHz linear transducer array (SL10-2, Supersonic Imagine). The diaphragm was scanned at the right zone of apposition, on the posterior axillary line vertical to the chest wall at the $9^{\text {th }}-11^{\text {th }}$ intercostal space. Minimal pressure was applied to the transducer in order to limit tissue deformation and modification of ventilatory mechanism. The whole ultrasound sequence is driven by using the research pack of 
the Aixplorer device (SonicLab, Supersonic Imagine, Aix en Provence, France). For plane wave experiments, the ultrasound sequence lasts $500 \mathrm{~ms}$ by acquiring 2500 images at $5000 \mathrm{~Hz}$ with no steering angle. For shear wave elastography, the supersonic shear imaging method [4] was used synchronized with an electromyographic device with a $2 \mathrm{~Hz}$ framerate.

In parallel to ultrasound acquisitions, subjects were studied in a semirecumbent position (40 degrees) with uncast abdomen, breathing through a mouthpiece while wearing a nose clip. The mouthpiece was connected to a two-way valve and pneumotachograph (3700 series, linearity range 0-160 L*min ${ }^{-1}$; Hans Rudolph, Kansas City, MO) for flow measurement. Mouth pressure (Pmo) was recorded using a differential transducer (model DP45-18, Validyne, Northridge, CA). Esophageal (Pes) and gastric (Pga) pressures were measured using $10-\mathrm{cm}$ balloon catheters, connected separately to differential pressure transducers (model DP45-32; Validyne, Northridge, CA) (Fig. 2). Electrical (DS7AH, Digitimer Ltd, Welwyn Garden City, England) and magnetic (Magstim 200, Magstim®, Whitland, United Kingdom) stimulators were used for nerve stimulation. Single pulse unilateral transcutaneous electrical phrenic nerve stimulation (PNS) or cervical magnetic stimulation (CMS) were setup to be delivered when the PWI sequence was triggered. PNS and CMS were delivered at functional residual capacity as estimated by slightly negative Pes. An overview of the setup is displayed in Figure 1 and Figure 2.

During the first experiment $(n=4)$, unilateral PNS and cervical magnetic stimulation (CMS) were used to elicit supramaximal and half-supramaximal responses in Pdi or electromyographic response during CMS and PNS, respectively. Displacements of the peritoneal membrane of the diaphragm and local displacements within the diaphragm were computed and compared to Pdi or electromyographic responses evoked by CMS or PNS, respectively.

During a second experiment $(n=14)$, participants performed a stepwise inspiratory loading protocol from 0 to $50 \%$ of maximal inspiratory pressure during closed-airways maneuvers and during ventilation against external elastic loads. Settings of the ultrasound scanner were defined as follow: B-mode enabled; dynamic range at $80 \mathrm{~dB}$; shear wave elastography mode enabled with penetration mode enabled. Gain and time gain compensation were tailored for each individual. Repeated measures correlation (R) were used to ascertain overall relationships variables.

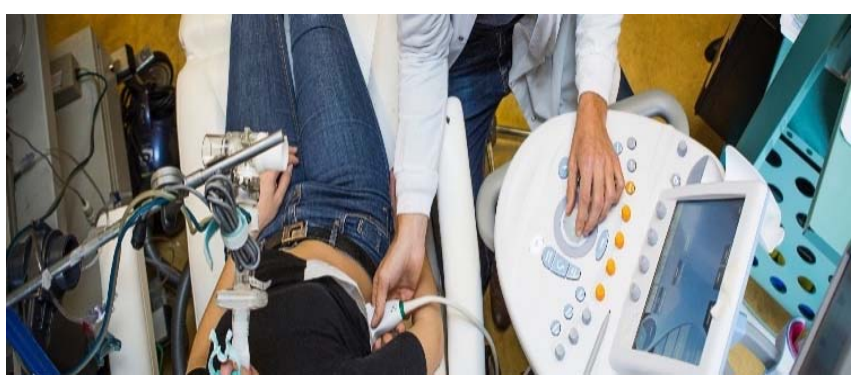

Figure 1: Overview of the experimental setup for acquisition of ultrasound and respiratory data.

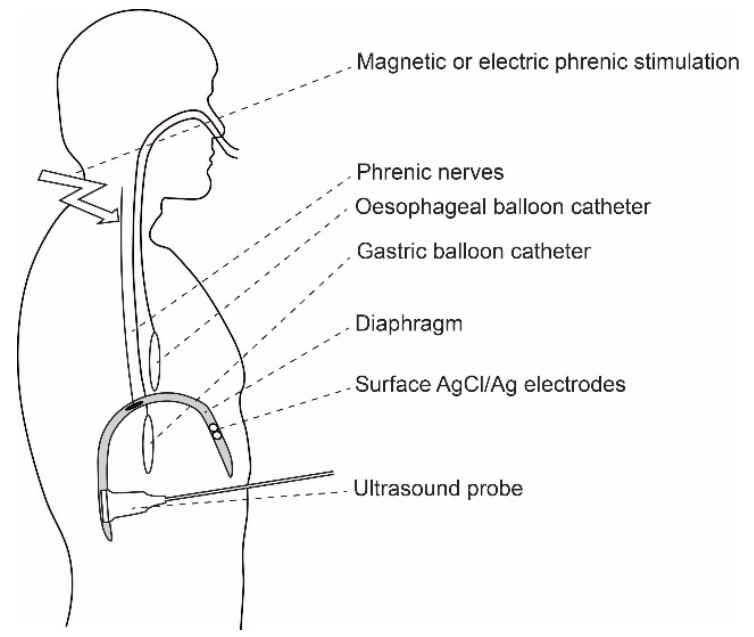

Figure 2: Positionning of the balloons and of the ultrasonic probe.

\section{ULTRAFAST PLANE WAVES FOR IMAGING DIAPHRAGM RESPONSE TO PHRENIC NERVE STIMULATION}

A typical image acquired using the PWI sequence is displayed in Figure 3 (45 mm depth x $38 \mathrm{~mm}$ width). From cross correlation of the successive images of the ultrafast movie, local axial displacements profiles were extracted. Typical displacements of the pleural and peritoneal diaphragmatic membranes as well as local axial displacements within the diaphragm during cervical magnetic stimulation and unilateral surface electrode stimulation of the phrenic nerve are displayed in Figure 4. Displacements evoked at low and high intensities were significantly different using both PNS and CMS $(0.45+/-$ $0.14 \mathrm{~mm}$ versus $1.38+/-0.58 \mathrm{~mm}$ and $0.40+/-0.29 \mathrm{~mm}$ versus $0.58+/-0.20 \mathrm{~mm}$, respectively).

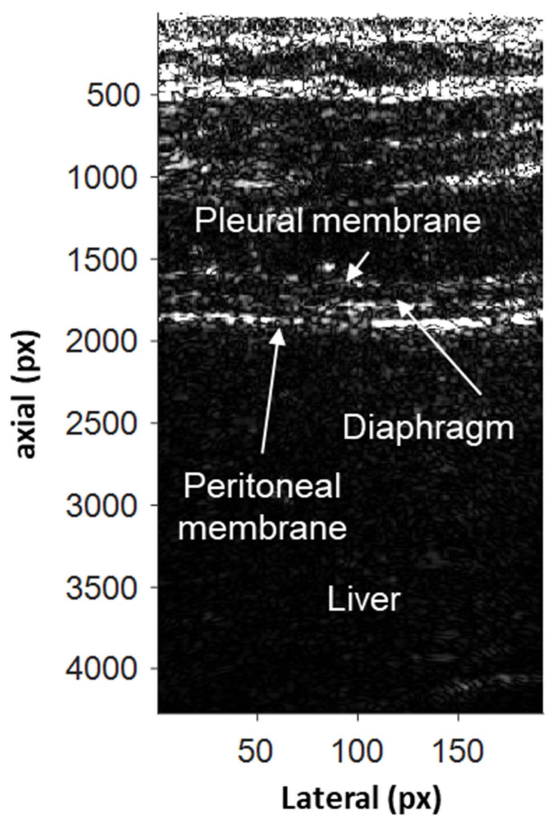

Figure 3: Typical image obtained using the plane wave imaging sequence with an intercostal approach at the right zone of apposition. 
A.

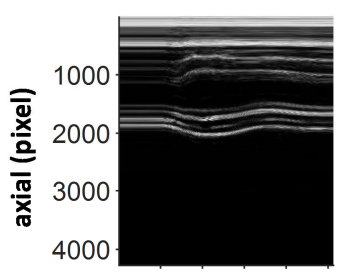

B.
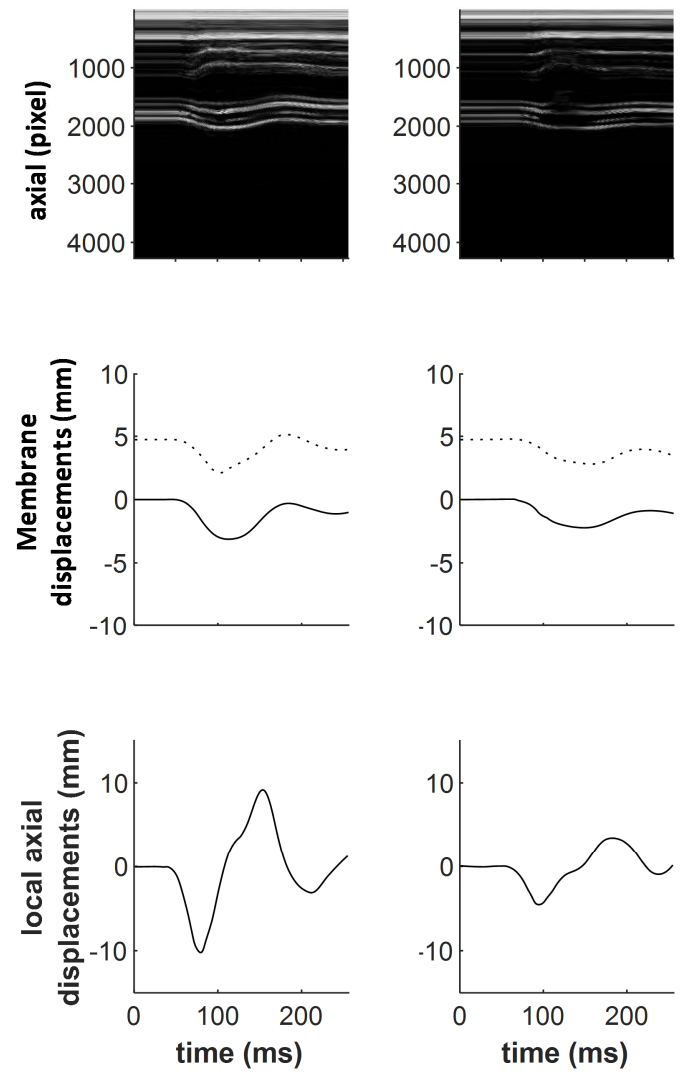

Figure 4: Time motion (upper pannel), displacements of the pleural (dotted line) and peritoneal (solid line) membranes (mid pannel), and local (i.e. within the diaphragm) lac axial displacements whithin the diaphragm (bottom panel) during supramaximal cervical magnetic stimulation (A.) and using a stimulation intensity half the transdiaphragmatic pressure response obtain with supramaximal stimulation (B.).

A typical elastogram of the diaphragm as acquired during the second experiment is displayed on Figure 5.

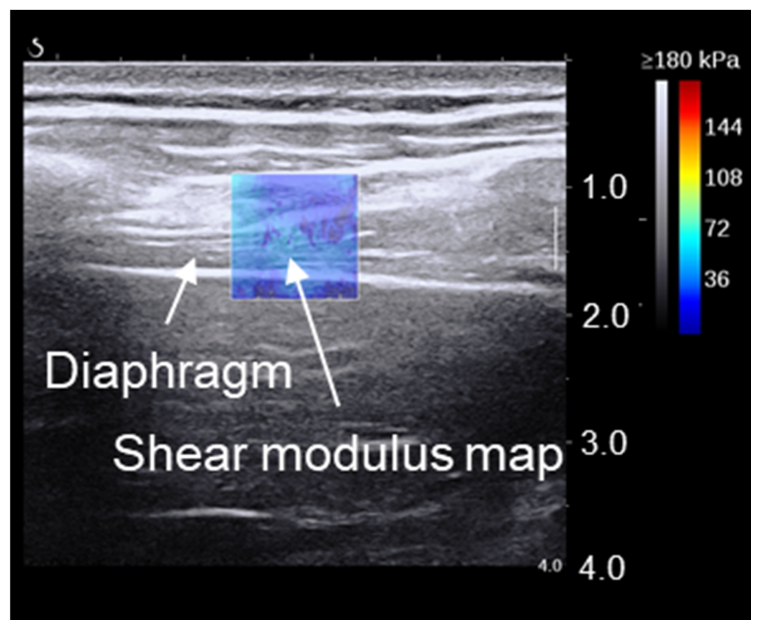

Figure 5: Measurement of diaphragm shear modulus at the right zone of apposition using ultrasound shear wave elastography.

The time behavior of shear modulus and transdiaphragmatique pressure during submaximal static inspiratory effort and ventilation against inspiratory loading is shown for one participant in Figure 6.
A.
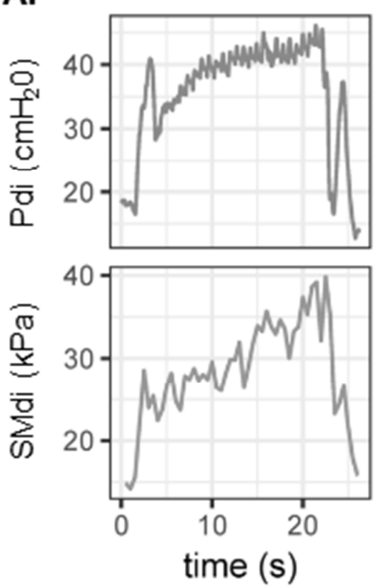

B.
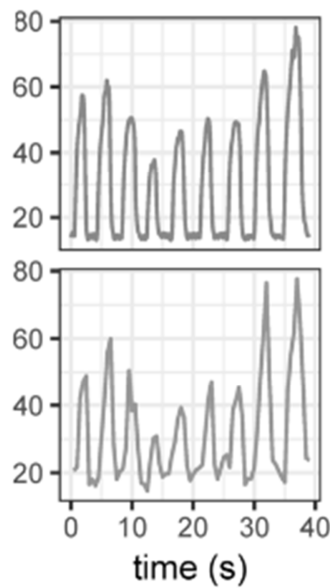

Figure 6: Typical recordings during submaximal static inspiratory efforts (A) and ventilation against inspiratory (B) loading in one participant. Pdi, transdiaphragmatic pressure, SMdi, diaphragm shear modulus.

Relationships between diaphragm shear modulus and transdiaphragmatic pressure for all participants is shown in Figure 7. During submaximal static inspiratory effort, mean Pdi was linearly related to mean SMdi $(\mathrm{R}=0.82$, 95\% CIs $[0.77, \quad 0.87], \quad \mathrm{p}<0.001)$. During inspiratory-loaded ventilation, Pdi (range: $1.26-125 \mathrm{cmH}^{2} \mathrm{O}$ ) swing correlated to maximal SMdi (range: $7.5-90.0 \mathrm{kPa}$ ) with $\mathrm{R}=0.69(95 \%$ CIs [0.66, 0.73], $\mathrm{p}<0.001)$.
A.

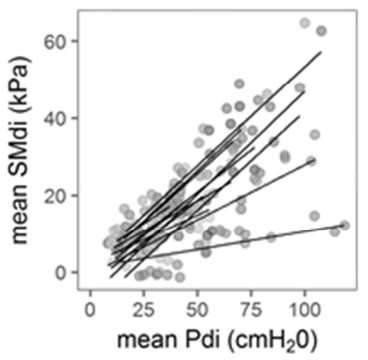

B.

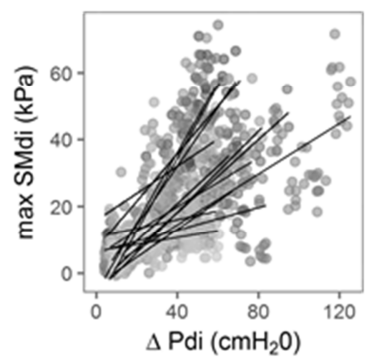

Figure 7: Relationship between transdiaphragmatic pressure and diaphragm shear modulus during submaximal static inspiratory efforts (A) and ventilation against inspiratory loading (B) for all participants. SMdi, diaphragm shear modulus; Pdi, variation of transdiaphragmatic pressure during the inspiratory time. Individual linear regression lines are shown.

\section{PERSPECTIVES}

The aim of the present study was to investigate the potential of PWI to image short responses ( 200 ms) evoked by artificial stimulation of the phrenic nerve(s) and the potential SWE to monitor change in diaphragm stiffness during continuous ventilation and/or respiratory maneuvers.

These preliminary data show that PWI may be used to discriminate large and small responses induced by artificial stimulation. Further research is needed to thoroughly investigate the relationship between PWI-based metrics and transdiaphragmatic pressure or electromyographic responses to artificial stimulation. This method have a strong potential for improving diagnosis comfort and specificity.

Our data also showed that changes in diaphragm shear modulus assessed using SWE reflected change in transdiaphragmatic pressure during both submaximal static inspiratory efforts and ventilation against inspiratory 
loading. As shown in Figure 7, diaphragm shear modulus may fail to increase along with transdiaphragmatic pressure. Misalignment of the ultrasound probe with diaphragm fascicles given the highly anisotropic nature of the skeletal muscle [5]. Methods to ensure high quality recordings of diaphragm shear modulus using SWE remain to be developed.

Both PWI and SWE grant new alternate methods for direct, noninvasive, and specific assessment of diaphragm contractility. Further methodological and technological developments are required to enable the use of these methods in patients

References

[1] S. American Thoracic Society/European Respiratory, "ATS/ERS Statement on respiratory muscle testing," Am J Respir Crit Care Med, vol. 166, no. 4, pp. 518-624, Aug 15, 2002.

[2] T. Deffieux, J. L. Gennisson, M. Tanter et al., "Assessment of the mechanical properties of the musculoskeletal system using 2-D and 3-D very high frame rate ultrasound," IEEE Trans Ultrason Ferroelectr Freq Control, vol. 55, no. 10, pp. 2177-90, Oct, 2008.

[3] F. Hug, K. Tucker, J. L. Gennisson et al., "Elastography for Muscle Biomechanics: Toward the Estimation of Individual Muscle Force," Exerc Sport Sci Rev, vol. 43, no. 3, pp. 125-33, Jul, 2015.

[4] Bercoff J, Tanter M, and Fink M. Supersonic shear imaging: a new technique for soft tissue elasticity mapping. IEEE Transactions on Ultrasonics Ferroelectrics and Frequency Control 51: 396-409, 2004.

[5] A. Sarwal, F. O. Walker, and M. S. Cartwright, "Neuromuscular ultrasound for evaluation of the diaphragm," Muscle Nerve, vol. 47, no. 3, pp. 31929, Mar, 2013. 